\title{
New approach to jitter reduction of an $x-$ ray streak camera in accumulation mode
}

\author{
J. Liu ${ }^{* a}$, A. G. MacPhee ${ }^{b}$, C. Liu ${ }^{a}$, B. Shan ${ }^{c}$, Z. Chang ${ }^{\mathrm{c}}$ and J. Wang ${ }^{\mathrm{a}}$ \\ ${ }^{a}$ Advanced Photon Source, Argonne National Laboratory, Argonne, IL 60439 \\ ${ }^{b}$ Lawrence Berkeley National Laboratory, Berkeley, CA 94720 \\ 'J. R. Macdonald Laboratory, Kansas State University, Manhattan, KS 66506
}

\begin{abstract}
ABSTRCT
An x-ray streak camera operating in accumulation mode was developed for studying ultrafast dynamics at synchrotron facilities. A laser-triggered photoconductive switch was used as a sweeping unit to obtain low timing jitter. The fast rise time of the ramp pulse generated by the switch $(90 \mathrm{ps})$ combined with the fast response of the traveling wave deflection plates (150 ps) significantly reduced the jitter caused by the shot-to-shot laser fluctuation. At $\sim 1 \%$ rms (root mean square) laser energy fluctuation, the resolution of the camera is 1.1 ps when over 5000 laser shots were accumulated. This is two times better than that of the previous design with slower response ( $300 \mathrm{ps})$ deflection plates.
\end{abstract}

Keywords: fast detector, streak camera, photoconductive switch, ultrashort laser, trigger jitter, synchronization

\section{INTRODUCTION}

X-rays from synchrotron radiation have recently been used as structural probes of solids in the ultrashort time domain. ${ }^{[1-}$

${ }^{3]}$ The pulse width of the x-ray from a third-generation light source, typically 50 to $100 \mathrm{ps,} \mathrm{is} \mathrm{limited} \mathrm{by} \mathrm{the} \mathrm{electron}$ bunch length in the storage ring. By combining such a long x-ray pulse with ultrafast x-ray detectors, it is possible to carry out ultrafast dynamic studies with a few picosecond or even subpicosecond time resolution. The time-resolved experiments can use a femtosecond laser as a pumping pulse, relatively long x-ray pulse from synchrotron radiation source as a probing pulse, and an x-ray streak camera as a fast detector. Accumulation of signals over thousands of laser and $\mathrm{x}$-ray shots is required due to low signal level. In this operating mode, the resolution of the streak camera is limited by the timing jitter from shot to shot.

The timing jitter of the commonly used avalanche transistor circuits for driving the deflection plates was on the order of tens of picosecond (rms). Therefore operating the streak camera in accumulating mode to achieve a few picoseconds time resolution is difficult. When a high-voltage photoconductive switch was first used on a streak camera as a sweeping circuit, ${ }^{[4]}$ it reduces the shot-to-shot jitter to a few picosecond ${ }^{[5,6]}$ Compared with the avalanche transistor circuit, the photo-conductive switches exhibit much lower jitter and are commonly referred to as "jitter-free" switches. The timing jitter for photoconductive switches is caused by the shot-to-shot laser pulse energy fluctuation. The jitter has been reduced to subpicosecond levels by improving the laser stability. ${ }^{[7]}$ To reduce the jitter even further, an extra GaAs switch can be used to compensate the sweep pulse amplitude resulting from laser intensity fluctuation. ${ }^{[8]}$ Realizing that the jitter is inversely proportional to the rise time of sweeping ramp voltage, we demonstrated that the timing jitter is significantly reduced by using a laser-triggered, fast GaAs sweeping unit and fast-response meander-type deflection plates. The measured time resolution of the streak camera is $1.1 \mathrm{ps}$ in accumulation mode with the laser RMS fluctuation being $\sim 1 \%$, 
which is significantly better than a similar camera with slower switch and deflection plates. ${ }^{[6]}$

\section{METHODS OF REDUCING TIMING JITTER}

The streak tube that was used in this work is similar to our previous design ${ }^{[9,10]}$ except that we redesigned the deflection plates. In the streak tube, the x-ray-produced electrons from the photocathode are accelerated by the high field (10 $\mathrm{kV} / \mathrm{mm}$ ) between the photocathode and the anode. The electrons then enter the region between a pair of deflection plates. The deflection plates are driven by a ramp voltage produced by a photoconductive switch that will be described later. Finally, the electrons are focused on the microchannel plate /phosphor screen by a magnetic lens. It is well known that the temporal resolution of streak camera are limited mainly by the transit time dispersion of the photoelectrons as they

travel from the photocathode to the deflection plates. It is also limited by the spatial resolution of the electron optics in the tube and by the sweeping speed. It was demonstrated before that the contribution from these two factors is about 0.5 ps for a previously developed camera. ${ }^{[10]}$

When the camera operates in the accumulation mode, the dominating factor that limits the time resolution is the timing jitter. While the response time of the photoconductive switch to the triggering laser may be instantaneous, the timing jitter is not zero unless the laser pulse is absolutely stable. At the present, the lasers used in synchrotron facilities for time-resolved x-ray studies are Ti:sapphire lasers operated at kilohertz repetition rate. The same laser is used to pump the sample under investigation and to trigger the photoconductive switch. The pulse energy fluctuation is on the order of $1 \%$ rms. This energy fluctuation causes the output amplitude of the photoconductor to change from shot to shot, which in turn produces timing jitter.

The timing jitter caused by laser fluctuation is illustrated in Figure 1, where the time dependence of the output amplitude of the switch from two laser shots is shown. We assume that the first pulse $\left(\mathrm{V}_{1}\right)$ has higher amplitude than the $\left(\mathrm{V}_{2}\right)$. If properly calibrated, it will take a longer time for the second pulse to reach a certain level of driving voltage (for example, at half of the averaged pulse high, $V_{a}$ ), which corresponds to the center of the phosphor screen. The time difference $\left(t_{2}-t_{1}\right)$ represents the time jitter due to the switch response to the fluctuation of laser intensity. The jitter of the switch can be simply expressed as

$$
\Delta t_{\text {jitter }}=0.5 T_{\text {rise }} \frac{\Delta V}{V_{a}} \text {. }
$$

Where $\mathrm{T}_{\text {rise }}$ is the rise time of sweeping pulse, $\Delta \mathrm{V} / \mathrm{V}_{\mathrm{a}}$ is the rms fluctuation in the shot-to-shot switch output voltage, which is related to the laser intensity fluctuation nonlinearly (if the switch is operated in a saturated mode to suppress the effect of the laser intensity fluctuation). Therefore, the jitter can be reduced by decreasing the rise time. 
With the previous camera, ${ }^{[6]}$ the rise time was limited to $300 \mathrm{ps}$ by the bandwidth of the deflection plates. The plates were redesigned so that the response time improved to $150 \mathrm{ps}$. The measured response time is shown in Figure 2. We expected the two-fold reduction in the response time would result in a two-fold reduction of the timing jitter.

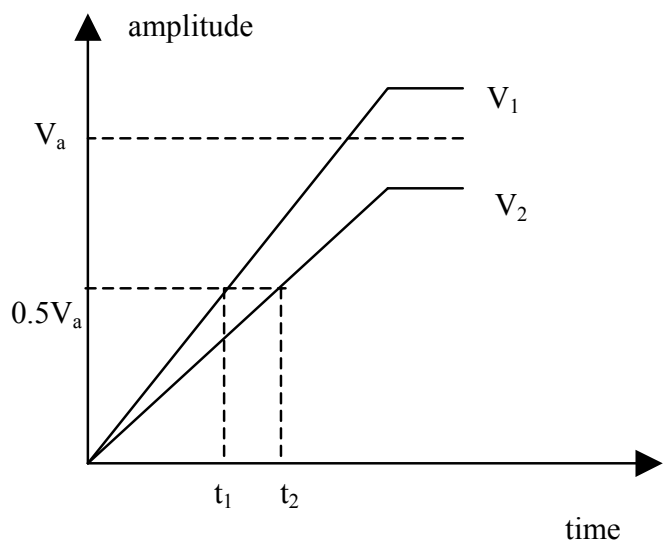

Figure 1. Illustration of timing jitter caused by amplitude fluctuation of sweeping pulse. $\mathrm{V}_{\mathrm{a}}$ is an averaging amplitude of sweeping pulses. $\Delta \mathrm{t}=\mathrm{t}_{2}-\mathrm{t}_{1}$ is the timing jitter.

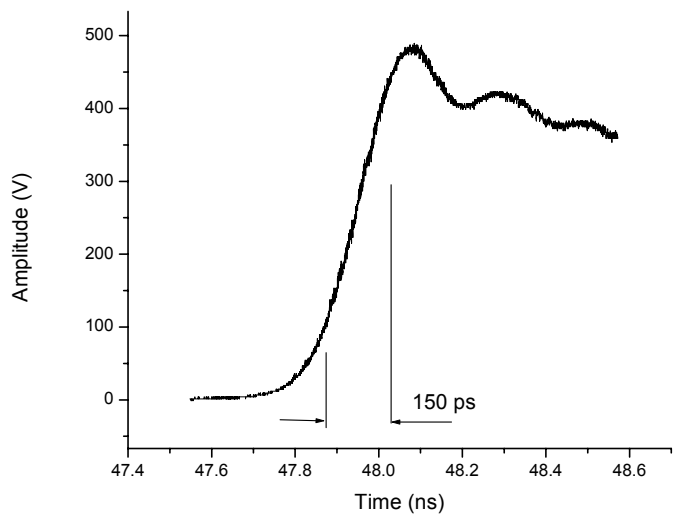

Figure 2. Response time (150 ps) of the newly designed deflection plates. It was measured by sending a pulse with $90 \mathrm{ps}$ rise time to the deflection plate. A $20 \mathrm{GHz}$ Tektronics sampling oscilloscope was used for the measurement.

\section{PHOTOCONDUCTIVE SWITCH}

The overall rise time of the field in the deflection plates is determined by the response time of the deflection plates and the photoconductive switch. It is desirable that the response time of the switch is much smaller than that of the deflection plates. The design of our switch is eased by the fact that the deflection sensitivity of our streak tube is high at $20 \mathrm{~cm} / \mathrm{kV}$. Only \pm 50 volts are needed to sweep electrons across a 20 -mm-diameter phosphor screen. The photoconductive switch was made from semi-insulate GaAs with a resistivity of $10^{7} \Omega-\mathrm{cm}$. The active area of GaAs is about $0.5 \mathrm{~cm}^{2}$. To reduce the contact resistance, two copper electrodes were deposited on the switch with a gap of $2 \mathrm{~mm}$ between them. In order to get a fast response time and reduce the pulse reflection, the switch is mounted on a circuit board with a $50 \Omega$ transmission line. Figure 3 shows a schematic diagram of the circuit. This design is much simpler and much easier to use than that of the ramp voltage generation with the two GaAs switch configuration. 


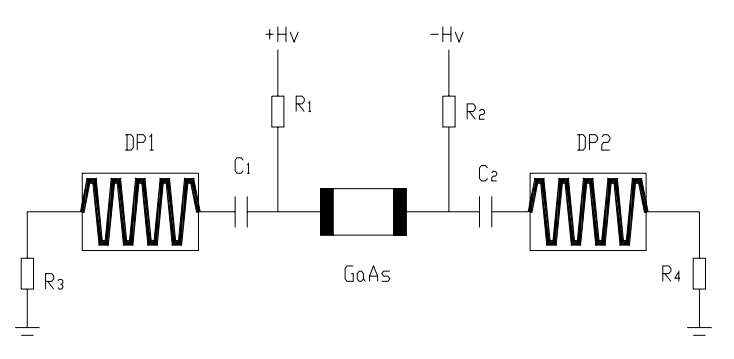

Figure 3. Schematic diagram the of photoconductive switch circuit. (DP1 and DP2 : deflection plates. $\mathrm{C}_{1}, \mathrm{C}_{2}$ : DC blocks; $\mathrm{R}_{3}, \mathrm{R}_{4}$ : matching resistors).

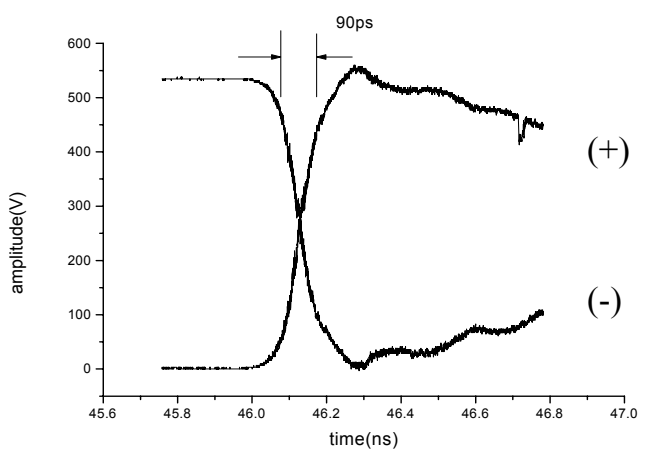

Figure 4. Symmetrical ramp pulse wave forms from the photoconductive switch with rise time 90 ps respectively, which used to drive the two arms of deflection plates.

With our design, the two pulses are generated by the same switch at the same time. The effect of laser energy fluctuation on the timing jitter is reduced when a photoconductive switch is operated in saturated mode at high laser illumination flux. Only $20 \mu \mathrm{J}$ laser energy is needed to saturate our photoconductive switch. As the laser flux increases, the peak of the output signal becomes more stable. However, the laser pulse flux can not be too high, since the pre-pulses and /or amplified spontaneous emission may start to distort the output of the switch or even damage the switch. Figure 4 shows the symmetrical ramp pulses produced by the switch measured by a TEK11801A Sampling Oscilloscope. The sweeping pulse rise time is $90 \mathrm{ps}$ with $500 \mathrm{~V}$ amplitude.

\section{MEASUREMENT OF TIMING JITTER}

The measurements were done using the newly established high-intensity laser facility at Kansas State University. A $25 \mathrm{~nm}$ gold layer coated on fused silica was used for the streak camera photocathode. The experimental setup is shown in Figure 5. Since the streak camera is also sensitive to uv light, we used ultrashort third-harmonic pulses to test the camera. The third-harmonic pulses of a Ti: sapphire laser are generated by focusing the $800 \mathrm{~nm}, 20 \mathrm{fs}, 1$ $\mathrm{mJ}, 1 \mathrm{kHz}$ laser pulses in air with an $\mathrm{f}=100 \mathrm{~mm}$ lens. A prism was used to separate the uv beam from the infrared beam. Shot-to-shot laser stability is about $1 \%$ evaluated by oscilloscope. A Michelson interferometer is used as a timing scale. One of the optical path lengths was varied to produce two pulses with known delay. The reflectivity of another arm's mirror is $95 \%$, which provides about $20 \mu \mathrm{J}$ laser energy to trigger the photoconductive switch. The GaAs photoconductor is triggered at $1 \mathrm{kHz}$ repetition rate. The delay time between the sweep pulses and the photoelectrons emitted by the photocathode can be adjusted continuously by moving the photoconductor switch on a rail. The streak images were integrated by a Liquid-Cooled Photometrics CCD camera (1024×1024 pixels). The static spatial resolution is about $120 \mu \mathrm{m}$ full width half maximum. The streaked images have been accumulated on the CCD camera with a CCD integration time of 5 seconds. Figure 6 shows the lineout of the streak image obtained with the 5000 shots. The FWHM of the plot is $1.1 \mathrm{ps}$ while the interval time between the two pulses is $3.3 \mathrm{ps}$. The sweep speed used in the calibration was $0.35 \mu \mathrm{m} / \mathrm{fs}$ on the CCD camera, comparable to the speed of light $(0.3$ $\mu \mathrm{m} / \mathrm{fs})$. The trigger jitter of the streak camera can be written as 


$$
\Delta t_{\text {jitter }}=\left[\left(\Delta t_{\text {meas }}\right)^{2}+\left(\Delta t_{s s}\right)^{2}\right]^{\frac{1}{2}}
$$

where $\Delta \mathrm{t}_{\text {meas }}$ is the measured time resolution under accumulation mode, which is $1.1 \mathrm{ps} . \Delta \mathrm{t}_{\mathrm{ss}}$ is the single-shot time resolution, $0.5 \mathrm{ps}$, that was measured before. ${ }^{[10]}$ Therefore the timing jitter is estimated to be $1 \mathrm{ps,} \mathrm{which} \mathrm{is} \mathrm{two} \mathrm{times}$ smaller than what was measured before with a lower response deflection system. ${ }^{[6]}$

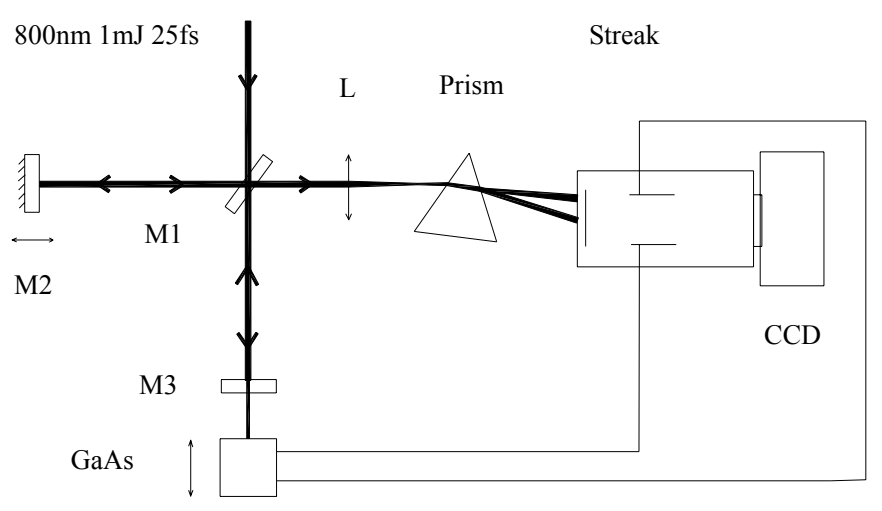

Figure 5. Experimental setup.

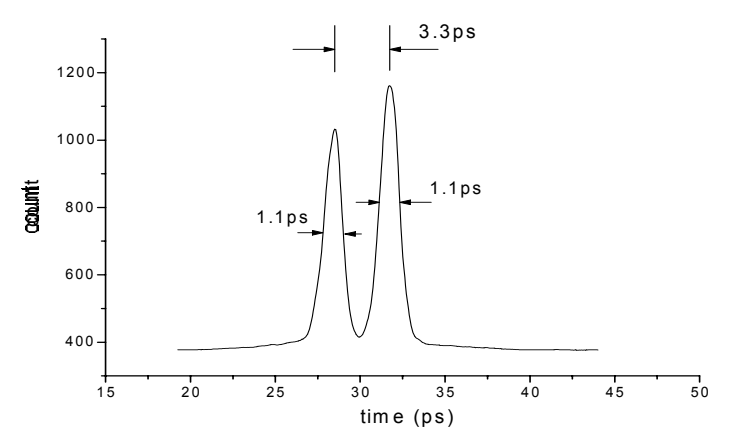

Figure 6. Measured time resolution of the streak camera under accumulation mode.

\section{CONCLUSION}

We have developed an x-ray streak camera driven by a photoconductive switch. We found that the timing jitter of the camera can be reduced significantly by reducing the rise time of the sweeping field. More specifically, the new 
deflection plates with $150 \mathrm{ps}$ response time resulted in a $1 \mathrm{ps}$ jitter in the streak camera at $1 \%$ laser fluctuation. Work is in progress to reduce the jitter to the femtosecond level by further improving the photoconductive switch and the deflection plates.

\section{ACKNOWLEDGMENTS}

This work is supported by the U.S. Department of Energy under contract no. W-31-109-ENG-38 and DE-FG0200ER15082.

\section{References:}

1. J. Larsson, P. A. Heimann, A. M. Lindenberg, P. J. Schuck, P. H. Bucksbaum, R. W. Lee, H. A. Padmore, J. S. Wark and R. W. Falcone, Appl. Phys. A66, pp. 587-591, 1998.

2. F. Raksi et al., J. Chem. Phys. 104, pp. 6066-6069, 1996.

3. A. M. Lindenberg, I. Kang, S. L. Johnson, T. Misalla, P. A. Heimann, Z. Chang, J. Larsson, P. H. Bucksbaum, H. C. Kapteyn, H. A. Padmore, R. W. Lee, J. S. Wark and R. W. Falcone, Phys.Rev.Lett. 84, pp.111-114, 2000.

4. W. Knox and G. Mourou, Opt. Commun. 37, pp. 203, 1981.

5. A. Maksimchuk, M. Kim, J. Workman, G. Korn, J. Squier, D. Du, M. Bouvier, D. Umstadter and G. Mourou, Rev. Sci. Instrum., 67, pp. 697-699, 1996.

6. J. Larsson et al., Optics Letters, 22, pp. 1012-1014, 1997.

7. G. A. Naylor, K. Scheidt, J. Larsson, M. Wulff and J. M. Filhol, Meas. Sci. Technol. 12, pp. 1858-1864, 2001.

8. C. Belzile, C. Y. Cote, T. Oksenhendler, D. Kaplan and J. C. Kieffer, Rev. Sci. Instrum. 73, pp. 1617-1620, 2002.

9. Z. Chang, A. Rundquist, J.Zhou, M. M. Murnane and H. C. Kapteyn, X. Liu, B.Shan, J. Liu, L.Niu, M. Gong and X. Zhang, Appl. Phys. Lett. 69, pp. 133-135, 1996.

10. Z. Chang et al., High Speed Photography and Photonics, SPIE 2869, pp. 971-975, 1996.

* contact: 1jinyuan@aps.anl.gov; phone 1630 252-5348; fax 16302529303 